PREHOSPITAL CARE

\title{
Prehospital cardiac arrest outcome is adversely associated with antiarrythmic agent use, but not associated with presenting complaint or medical history
}

\author{
R B Vukmir, and the Sodium Bicarbonate Study Group
}

Emerg Med J 2004;21:95-98

Study objective: This study associated survival from prehospital cardiac arrest to patient historical variables including presenting complaint, medications used, and medical history as a secondary end point in a trial evaluating the effect of bicarbonate administration. This raises issues concerning extensive prehospital historical assessment that may potentially delay care and transport.

Correspondence to:

R B Vukmir, Medical Director, Emergency Consultants, Inc, University of Pittsburgh Medical Center Northwest, Department of Emergency Medicine, and Safar Center for Resuscitation Research, One Spruce Street, Franklin, PA 16323, USA; rbv@csonline.net

Accepted for publication 21 September 2003
Methods: This prospective multicentre trial enrolled 874 prehospital cardiac arrest patients encountered by urban, suburban, and rural emergency medical services. This group underwent conventional ACLS intervention followed by empiric early administration of sodium bicarbonate $(1 \mathrm{mEq} / \mathrm{l})$. Survival was measured as the presence of vital signs on emergency department arrival. Data analysis used Student's $t$ test, Fisher's exact test, $\chi^{2}$ with Pearson correlation, and logistic regression $(p<0.05)$.

Secondary end points were analysed including an association with common historical variables such as medical history, presenting complaint, or drugs used.

Results: The overall survival rate was $13.9 \%$ (110 of 793) of prehospital arrest patients. There was no correlation between historical factors, such as chief complaint or history of present illness $(p=0.277)$, medical history $(p=0.425)$, presence of specific disease conditions $(p=0.1125-0.956)$, or overall drug use $(p=0.002-0.9848)$. However, there was an adverse association between specific antiarrhythmic use $(p=0.003)$ and outcome.

Conclusion: There is little relation of patient historical factors on the outcome from prehospital cardiac arrest raising issues of efficiency with history taking in prehospital care and transport.
$\mathrm{T}$ he use of prehospital healthcare providers to intervene in acute cardiac emergencies has historically been a focus of emergency care. However, Dean reported on the outcome of 134 patients who received mobile paramedic unit care compared with control patients without paramedic intervention demonstrating no change in outcome by multiple logistic regression analysis. ${ }^{1}$ Defibrillation was the only beneficial intervention identified, but also added a 29 minute delay to hospital arrival suggesting the need for more streamlined care.

Later, Shuster went on to evaluate 15 prehospital studies during the early years of emergency medical care suggesting no benefit of prehospital administration of any of a number of commonly administered prehospital drugs. ${ }^{2}$ Qualitatively, there have been few studies that have examined the use of such agents as albuterol, bicarbonate, bronchodilator agents, diazepam, dobutamine, dopamine, glucose, isoproterenol, naloxone, or nitrous oxide for their prehospital efficacy. ${ }^{3}$

Paramedic effectiveness has been described for advanced cardiac life support (ACLS) intervention with a 91.7\% success rate of obtaining intravenous access and $91 \%$ for intubation; however, drug administration was only consistent with $43 \%$ of resuscitation recommendations by intravenous route and $37 \%$ by endotracheal route. ${ }^{4}$ Stricter compliance with national ACLS guidelines facilitation involving refresher training courses may improve effectiveness.

Four factors are related to the ability to resuscitate patients in prehospital arrest: time to starting rescue procedures, use of electrical defibrillation, accuracy of technique of basic life support (BLS), and ventilation efficacy, decreasing in utility. ${ }^{4}$

The "early defibrillation" controversy has once again raised interest in the use of first responders or emergency medical technicians (EMTs) in a two tier response system. Wilson evaluated 126 patients whose care was limited to BLS: mask oxygen, intravenous fluids, closed chest massage, and artificial respiration. ${ }^{5}$ The survival rate was $22 \%$ (28) to hospital admission and 9\% (11) to hospital discharge, with a favourable prognosis group identified to include those with initial rhythm of ventricular fibrillation or tachycardia, $14 \%$ ( 7 of 50); and initial blood pressure $>90 \mathrm{~mm} \mathrm{Hg}$ and pulse rate $>50 \mathrm{bpm}, 50 \%$ ( 3 of 6 ). However, if the patient was in cardiac arrest, then CPR did not change outcome regardless.

We now may be at a point in the emergency medical services (EMS) realm when efficiency needs may mandate more rapid assessment in the field.

\section{METHODS}

This prospective, randomised, multicentre clinical trial involved cardiac arrest patients encountered by paramedics in a prehospital setting, and transported to hospitals within the study area, usually within a 5-30 minute transport radius. The multicentre trial enrolled patients encountered by Western Pennsylvania EMS systems into this protocol. The primary end point was survival and the secondary end point was correlation to historical variables.

Inclusion criteria were subjects suffering from cardiac arrest refractory to defibrillation in whom intravenous access was obtained. Exclusion criteria included those subjects suffering from overt respiratory arrest or traumatic, children $(<18$ years $)$ and those without intravenous access. Patients

\footnotetext{
Abbreviations: EMS, emergency medical services; ED, emergency department; ACLS, advanced cardiac life support; BLS, basic life support; EMT, emergency medical technician
} 
received standard ACLS, protocol including chest compressions, ventilation, defibrillation, adrenaline (epinephrine) $(0.01 \mathrm{mg} / \mathrm{kg})$, atropine $(0.01 \mathrm{mg} / \mathrm{kg})$, and antiarrhythmics or pressor agents as warranted. Patients were randomised to a treatment group receiving empiric dose of bicarbonate (Abbott, USA) 1 ample $(50 \mathrm{mEq} / \mathrm{l})$ early in the arrest cycle. The control group received an equal amount of normal saline in a double blinded fashion to clarify the benefits of the osmolar load compared with base deficit correction.

The historical information was collected by EMS personnel in routine fashion, and data abstracted by implicit review of prospective datasheets.

Routine demographic and clinical variables related to outcome were analysed including demographics, response to bicarbonate administration, scene factors, response time, cardiopulmonary variables, procedures, and duration of arrest (box). Historical factors including history of present illness such as chest, abdomen pain and dyspnoea; medical history such as myocardial infarction, coronary heart failure, or chronic obstructive pulmonary disease; and antecedent use of antiarrhythmic medicines including procainamide, quinidine, and mexiletine. Routine cardiopulmonary variables were monitored. Neurological outcome was measured initially as the Glasgow Coma Score, while long term outcome was assessed by the Folstein Mini Mental Exam after arrest. $^{67}$ Patient outcome was recorded as the return of spontaneous circulation (mean atrial pressure of $50 \mathrm{~mm} \mathrm{Hg}$ ) and initial emergency department survival (discharge) as a primary end point.

Patients were enrolled under the Doctrine of Implied Consent for the emergency use of an accepted resuscitation modality and notification was provided if requested by family or healthcare resources. In addition, administration of an FDA approved agent (sodium bicarbonate) in the emergency setting for moderate to prolonged arrest may be the standard of care, and in conjunction with the above conditions that are met, consent could be waived. This study, was approved by the University of Pittsburgh Institutional Review Board under this rationale and was modified to address Office for Protection from Research Risk issues concerning "deferred consent"s

Numerical data were represented as means and standard deviation with Student's $t$ test, Fisher's exact test, $\chi^{2}$ with Pearson correlation tests uded for logistic regression intergroup comparison $(\alpha<0.05)$ (SPSS/PC+, Chicago, IL). The study results were examined by the investigators at three month intervals (or $25 \%$ of projected patients) to verify early trends and outcome with capability of later modification.

The sample size of 1000 was sufficient to delineate a $50 \%$ difference in survival and neurological outcome at $80 \%$ power and 95\% confidence intervals between control and treatment groups. This estimate was based on a $12 \%$ rate of return of spontaneous circulation (ROSC) in the city of Pittsburgh prehospital arrests.

\section{RESULTS}

The overall survival rate was $13.9 \%$ (110 of 793) of prehospital cardiac arrest patients. (fig 1).

There was no association between historical factors-the presence of chest pain, abdominal pain, shortness of breath, mental status change, to cardiac arrest survival $(p=0.276)$ (table 1).

There was no association between medical historyspecifically disease conditions such as myocardial infarction, congestive heart failure, chronic obstructive pulmonary disease, hypertension, diabetes mellitus, cancer, cerebrovascular accident, coronary artery bypass graft, or transplant $(\mathrm{p}=0.1125-0.956)($ table 2$)$.
Prehospital variables correlated to survival

- Patient characteristics

- Age, weight, sex

- Response time

- ET Arrest, ET ByCPR, ET BLS, ET ACLS, ET ROSC, ET Hosp

- Interventions

- Bicarbonate (dose, weight based)

- Scene factor

- Bystander CPR, witnessed

- Cardiopulmonary variables

- Initial rhythm, initial systolic blood pressure (ISBP), IDBP

- Procedures

- Intubation, IV, other

- Duration of arrest

- Short $(<5 \mathrm{~min})$, moderate $(5-15 \mathrm{~min})$, long term (>15 min)

- EMS coverage

- Urban, suburban, rural

- Medical history

- MI, HTN, DM, CHF, COPD, CABG

- Drug

- Cardiac, HTN, arrhythmia, pulmonary, haematological, Gl, psychiatric, seizure

However, there was an association between taking antiarrhythmic drugs $(p=0.003)$ and decreased survival in the prehospital cardiac arrest patient (table 3 ). In addition, there was an adverse trend noted with gastrointestinal medicines $(\mathrm{p}=0.085)$.

There was no association between drugs such as other cardiac, antihypertensives, pulmonary, haematological, gastrointestinal, psychiatric or antiseizure agents (table 4).

\section{DISCUSSION}

Brison's demographic analysis of the cardiac resuscitation experience of 1510 cardiac arrest patients where $92.1 \%$ of

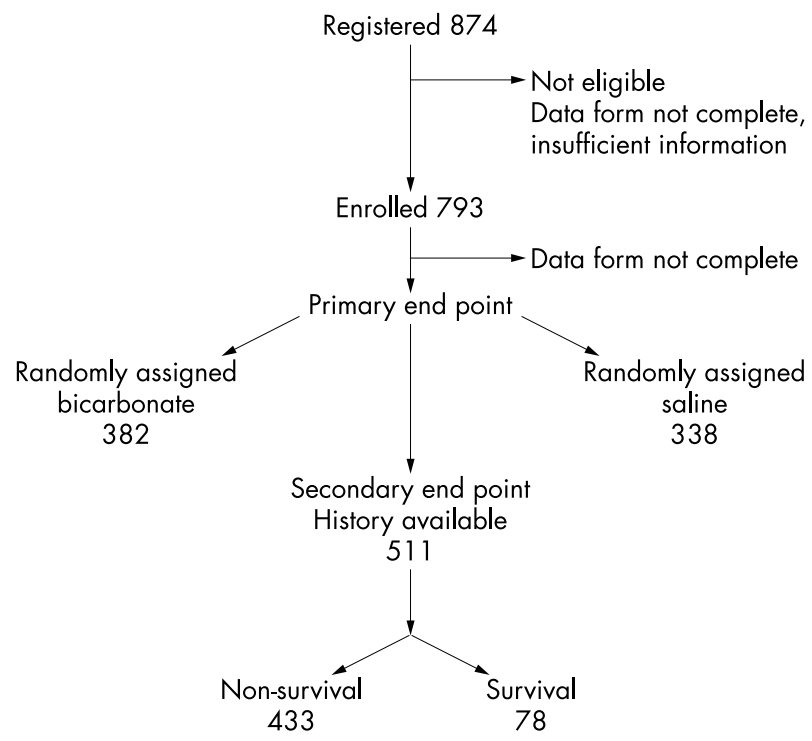

Figure 1 Trial profile. 
Table 1 History of patient illness correlated to survival

\begin{tabular}{|c|c|c|c|c|}
\hline & No & Yes & Total & \\
\hline \multirow[t]{3}{*}{ Chest pain } & 40 & 8 & 48 & \\
\hline & 83.3 & 16.7 & 9.4 & \\
\hline & 9.2 & 10.3 & & \\
\hline \multirow[t]{3}{*}{ Abdominal pain } & 18 & 1 & 19 & \\
\hline & 94.7 & 5.3 & 3.7 & \\
\hline & 4.2 & 1.3 & & \\
\hline \multirow[t]{3}{*}{ SOB } & 27 & 5 & 32 & \\
\hline & 84.4 & 15.6 & 6.3 & \\
\hline & 6.2 & 6.4 & & \\
\hline \multirow[t]{3}{*}{ Mental status change } & 25 & 9 & 34 & \\
\hline & 73.5 & 26.5 & 6.7 & \\
\hline & 5.8 & 11.5 & & \\
\hline \multirow[t]{3}{*}{ Other } & 41 & 13 & 54 & \\
\hline & 75.9 & 54.1 & 10.6 & \\
\hline & 9.5 & 11.7 & & \\
\hline \multirow[t]{3}{*}{ Cardiac arrest } & 243 & 37 & 280 & \\
\hline & 86.8 & 13.2 & 54.8 & \\
\hline & 56.1 & 47.4 & & \\
\hline \multirow[t]{5}{*}{ Unknown } & 36 & 5 & 41 & \\
\hline & 87.8 & 12.2 & 8.0 & \\
\hline & 8.3 & 6.4 & & \\
\hline & 433 & 78 & 511 & $\chi^{2}$ Pearson correlation \\
\hline & 84.7 & 15.3 & 100.0 & $p=0.27675$ \\
\hline
\end{tabular}

patients were 50 years of age, $68.3 \%$ were male and $79.6 \%$ of arrests occurred at home. ${ }^{9}$ The average ambulance response time of witnessed events was 7.8 minutes with an overall survival rate of $2.5 \%$. Factors predicting survival include age, ambulance response time, whether CPR started before ambulance arrival, but interestingly was not related to early defibrillation.

Tresch evaluated a population of 381 cardiac arrest patients comparing older and younger ( $<20$ years) cohorts, who have undergone paramedic witnessed cardiac arrest. ${ }^{10}$ The elderly patient cohort more commonly had a past history of heart failure $(25 \% \vee 10 \%)$, was more commonly taking digoxin $(40 \% \vee 20 \%)$, diuretics (35\% v25\%), and was more likely to complain of dyspnoea ( $53 \% \vee 40 \%)$. Younger patients were more likely to complain of chest pain (27\% v 13\%) and presented in ventricular fibrillation $(42 \% \quad v \quad 22 \%)$. Interestingly, the patients' chief complaint correlated with initial rhythm where $68 \%$ of those with chest pain demonstrated a ventricular fibrillation event compared with $21 \%$ of those with dyspnoea. Although, there were equivalent initial resuscitation rates in the elderly their survival to discharge was decreased comparatively (24\% to $10 \%$ ).

Survey data offered by $\mathrm{Ng}$ concerning 105 younger arrests (1-39 years) patients found a male predominance (62\%), secondary to cardiac disease $(38 \%)$ attributable to arthrosclerotic heart disease in $50 \%$, and secondary toxic exposure in $21 \%{ }^{11}$ The most common presenting rhythm was ventricular fibrillation (VF) (45\%) associated with a $48 \%$ resuscitation rate with over $28 \%$ of post-resuscitation patients

\begin{tabular}{ll} 
Table 2 & Medical history correlated to survival \\
\hline Disease condition & Significance $(\mathbf{p})$ \\
\hline Ml & 0.2092 \\
CHF & 0.3808 \\
COPD & 0.8726 \\
HTN & 0.9556 \\
DM & 0.75149 \\
Cancer & 0.8534 \\
CVA & 0.1921 \\
CABG & 0.3939 \\
Transplant & 0.1125 \\
\hline
\end{tabular}

Table 3 Drug type correlated to survival

\begin{tabular}{ll}
\hline Drug & Significance $(\mathbf{p})$ \\
\hline Cardiac & 0.9848 \\
HTN & 0.8608 \\
Arrhythmia & 0.0025 \\
Pulmonary & 0.9554 \\
Haematological & 0.3492 \\
Gl & 0.0846 \\
Psychiatric & 0.3930 \\
Seizure & 0.5249 \\
\hline
\end{tabular}

progressing to long term survival. Favourable outcome was predicted by the arrest being witnessed, or associated with primary cardiac arrhythmia; while asystole was a negative prognostic indicator and age, sex, race, bystander CPR, and paramedic response time were not significant prognostic factors affecting long term survival.

Clearly, there are widely discrepant rates of survival in hospital compared with prehospital cardiac arrest events. Rosenberg evaluated 300 hospitalised patients demonstrating a $54 \%$ initial post-CPR survival followed by $23 \%$ survival to hospital discharge. ${ }^{12}$ Predictors of good resuscitation outcome include an initial ventricular tachycardia or fibrillation rhythm, and brief duration of $\mathrm{CPR}<30$ minutes.

Bonnin evaluated a 181 patient group where 6\% (10) who failed prehospital resuscitation survived to hospitalisation, but only $0.6 \%$ (1) was discharged neurologically intact; with gender as the only predictive correlate. ${ }^{13}$ Van der Hoeven conducted a retrospective chart review of 309 adult patients where $13.6 \%$ survived to hospital discharge with favourable prognosis associated with the event being witnessed at time of arrest, short call response interval, initial cardiac rhythm of VF or VT and the provision of appropriate ACLS care. ${ }^{14}$ Improvement of all aspects of the "prehospital chain of survival" is likely to result in better outcome.

The outcome of prehospital cardiac arrest has been adversely affected by significant patient comorbidity. Hallstrom demonstrated a correlate between the comorbidity

Table 4 Arrythmia drugs correlated to emergency room survival

\begin{tabular}{|c|c|c|c|c|c|}
\hline \multirow{2}{*}{$\begin{array}{l}\text { Arrhythmia } \\
\text { drug }\end{array}$} & & \multicolumn{4}{|l|}{ ER survival } \\
\hline & & Non-survival & Survival & & \\
\hline \multirow[t]{3}{*}{ None } & (n) & 373 & 55 & 428 & \\
\hline & (\%) & 87.1 & 12.9 & 64.3 & \\
\hline & (\%) & 8.0 & 59.8 & & \\
\hline \multirow[t]{3}{*}{ Unknown } & & 196 & 32 & 228 & \\
\hline & & 86.0 & 14.0 & 34.2 & \\
\hline & & 34.1 & 34.8 & & \\
\hline \multicolumn{6}{|l|}{ Specific } \\
\hline \multirow[t]{3}{*}{ Procainamide } & & 2 & 2 & 4 & \\
\hline & & 50.0 & 50.0 & 6 & \\
\hline & & 0.3 & 2.2 & & \\
\hline \multirow[t]{3}{*}{ Quinidine } & & 1 & 2 & 3 & \\
\hline & & 33.3 & 66.7 & 0.5 & \\
\hline & & 0.2 & 2.2 & & \\
\hline \multirow[t]{2}{*}{ Mexiletine } & & & 1 & 1 & \\
\hline & & & 100.0 & 2 & \\
\hline \multirow{5}{*}{ Disopyramide } & & 2 & & 2 & \\
\hline & & 100.0 & & 0.3 & \\
\hline & & 0.3 & & & \\
\hline & & 514 & 92 & 666 & \\
\hline & & 86.2 & 13.8 & 100 & \\
\hline $\begin{array}{l}\chi^{2} \text { Pearson } \\
\text { correlation }\end{array}$ & & & & & $p=0.00255$ \\
\hline
\end{tabular}


index featuring such historical factors such as congestive heart failure $(p=0.004)$ in 282 out of hospital arrest victims.

The question of successful resuscitation in critical care units assumes importance because patients often have multisystem disorders often responsible for poor outcome after CPR. Peterson reviewed the records of 114 MICU patients with $70 \%$ mortality, $18 \%$ successfully resuscitated but died before discharge while $11 \%$ of patients survived to discharge. ${ }^{15}$ Pre-arrest conditions including hypotension, sepsis or APACHE II Acute Physiology Score increase and arrest conditions of duration of resuscitation effort are independently associated with poor outcome after CPR. Therefore, CPR can be successful in ICU patients and both pre-arrest and arrest variables can be predictive of outcome.

Initially, in our evaluation a 350 patient group both the presence of coronary artery bypass graft procedure $(p=0.05)$ and a prior cerebrovascular event were associated with adverse outcome. The propensity to make a type I $(\alpha)$ error to falsely conclude that a significant difference exists between population, when in fact there is none is hopefully mitigated by the increase in sample size from 350 to 874 patients with an accompanying increase in study power.

We found no association between chief complaint, historical features, or drugs used. An extensive amount of time is spent in the prehospital realm exploring these aspects of patient care and perhaps the time would be more appropriately directed elsewhere in the emergency care continuum. We have spanned the continuum from the use of standing orders to reduce scene time in medical cases, load and go strategies for trauma to the diagnostic use of prehospital 12 lead ECGs, which may actually prolong scene time. ${ }^{16} 17$

There are smaller studies that suggest an adverse outcome based on such premorbid factors. Therefore proper study design with a focused end point may prove helpful to further elucidate this issue in the prehospital realm.

\section{ACKNOWLEDGEMENTS}

We would like to thank Lisa Dotterweich and Nina Tulac for assistance with data analysis as well as Christine Henderson and Melodie Braden for manuscript preparation.

Funding: the work was supported in part by The Laerdal Foundation for Acute Medicine and University of Pittsburgh Competitive Medical Research Fund.

Conflicts of interest: none declared.

\section{SODIUM BICARBONATE STUDY GROUP}

Primary investigator: Rade B Vuikmir MD. Beaver: Paul Peindl MD; Charles Bowers EMT-P; Joseph Campbell EMT-P. Greensburg: John Reed MD; William Groft EMT-P. Indiana: Steve Bashor MD; James Dixon MD; BJ Pino EMT-P. McKees Rocks: Peter Stevenson MD; Anthony Sanvito EMT-P. Monessen: Frank Hertzog MD; Bill Hess EMT-P; Mark Sellew EMT-P; Mark Eglgi EMT-P. Monroeville: Dave Natale MD; Doug Nicole EMT-P. Pittsburgh: Laurence Katz MD; Michael Sullivan MD; Uwe Ebmeyer MD; Nicholas Bircher MD; Paul Paris MD; James Menegazzi Ph.D; Peter Safar MD. Sewickley: J Nagan MD; J Withee EMT-P.

\section{REFERENCES}

1 Dean NC, Haug PJ, Hawker PJ. Effect of mobile paramedic units on outcome in patients with myocardial infarction. Ann Emerg Med 1988;17:61-8.

2 Shuster M, Chong J. Pharmacological intervention in prehospital care, a critical appraisal. Ann Emerg Med 1989;18:126-30.

3 Atkins JM. Emergency medical services systems in acute cardiac are state of the art. Circulation 1986;74:4-8.

4 Hodgetts TJ, Brown T, Driscoll P, et al. Pre-hospital cardiac arrest: room for improvement. Resuscitation 1995;29:47-54.

5 Wilson BH, Severance HW, Raney MP, et al. Out of hospital management of cardiac arrest by basic emergency medical technicians. Am J Cardiol 1984;53:68-70.

6 Teasdale G, Jennet B. Assessment of coma and impaired consciousness: a practical scale. Lancet 1974;ii:81-4.

7 Folstein M, Folstein S. Mini-mental status: A practical method for grading the cognitive state of patient for the clinician. J Psychiat Res 1975; 12:189-98.

8 Abramson NS, Safar P. Deferred consent: use in clinical resuscitation research. Brain resuscitation clinical trial II study group. Ann Emerg Med 1990;19:781-4.

9 Brison RJ, Davidson JR, Dreyer JF, et al. Cardiac arrest in Ontario: circumstances, community response, role of prehospital defibrillation, and predictors of survival. Can Med Assoc J 1992;147:191-9.

10 Tresch DD, Thakur RK, Hoffmann RG, et al. Comparison of outcome of paramedic witnessed cardiac arrest in patients younger and older than 70 years. Am J Cardiol 1990;65:453-7.

$11 \mathrm{Ng} \mathrm{AY}$, Clinton JE, Peterson G. Nontraumatic prehospital cardiac arrest ages 1-39 years. Am J Emerg Med 1990;8:87-91.

12 Rosenberg M, Wang C, Hoffman-Wilde S, et al. Results of cardiopulmonary resuscitation. Arch Intern Med 1993;153:1370-5.

13 Bonnin MJ, Swor RA. Outcomes in unsuccessful field resuscitation attempts. Ann Emerg Med 1989;18:507-12.

14 van der Hoeven JG, Waanders H, Compier EA, et al. Evaluation of an emergency medical system. The prognosis in patients with an out of hospital cardiac arrest. Neth J Med 1994;44:5-11.

15 Peterson MW, Geist $\amalg$, Schwartz DA, et al. Outcome after cardiopulmonary resuscitation in a medical intensive care unit. Chest 1991;100:168-74.

16 Pointer JE, Osur MA. Effects of standing orders on field times. Ann Emerg Med 1989;18:1119-21.

17 Aufderheide TP, Hendley GE, Thakur RK, et al. The diagnostic impact of prehospital 12 lead electrocardiography. Ann Emerg Med 1990;19:1280-7. 